\title{
Coulomb-Assisted Dissociative Electron Attachment: Application to a Model Peptide
}

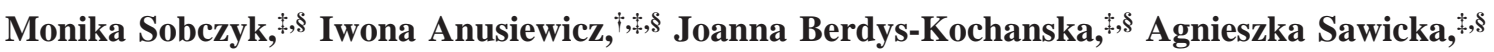 \\ Piotr Skurski,,$\$$, and Jack Simons, ${ }^{*, \hbar}$
}

Chemistry Department and Henry Eyring Center for Theoretical Chemistry, University of Utah, Salt Lake City, Utah 84112, U.S.A., and Department of Chemistry, University of Gdansk, 80-952 Gdansk, Poland

Received: August 16, 2004; In Final Form: October 12, 2004

\begin{abstract}
The fragmentation of positively charged gas-phase samples of peptides is used to infer the primary structure of such molecules. In electron capture dissociation (ECD) experiments, very low-energy electrons attach to the sample and rupture bonds to effect the fragmentation. It turns out that ECD fragmentation tends to produce cleavage of very specific types of bonds. In earlier works by this group, it has been suggested that the presence of positive charges produces stabilizing Coulomb potentials that allow low-energy electrons to exothermically attach to $\sigma^{*}$ orbitals of certain bonds and thus to cleave those bonds. In the present effort, the stabilizing effects of Coulomb potentials due to proximal positive charges are examined for a small model peptide molecule that contains a wide range of bond types. Direct attachment of an electron to the $\sigma^{*}$ orbitals of eight different bonds as well as indirect $\sigma$ bond cleavage, in which an electron first binds to a carbonyl $\mathrm{C}=\mathrm{O} \pi^{*}$ orbital, are examined using ab initio methods. It is found that direct attachment to and subsequent cleavage of any of the eight $\sigma$ bonds is not likely except for highly positively charged samples. It is also found that attachment to a $\mathrm{C}=\mathrm{O} \pi^{*}$ orbital followed by cleavage of the nitrogen-to- $\alpha$-carbon bond is the most likely outcome. Interestingly, this bond cleavage is the one that is seen most commonly in ECD experiments. So, the results presented here seem to offer good insight into one aspect of the ECD process, and they provide a means by which one can estimate (on the basis of a simple Coulomb energy formula) which bonds may be susceptible to cleavage by low-energy electron attachment.
\end{abstract}

\section{Introduction}

We recently showed ${ }^{1}$ that low-energy electrons (i.e., with kinetic energies near zero) could directly and even vertically (i.e., at the equilibrium geometry of the neutral) attach to and subsequently fragment $\mathrm{S}-\mathrm{S} \sigma$ bonds in disulfide-linked dimers of Ac-Cys-Ala ${ }_{n}$-Lys (with $n=10,15$, and 20) that are protonated at their two Lys sites. An example of such a species is shown in Figure 1 where the alanine helices are shown in red, the cystine linkage containing the $S-S$ bond appears in the center, and the two Lys sites are at the termini.

In the mechanism treated in ref 1 , an electron enters the $S-S$ antibonding $\sigma^{*}$ orbital to form a metastable anion that can either undergo electron autodetachment at a rate of ca. $10^{15}-10^{14} \mathrm{~s}^{-1}$ or fragment (promptly because of the repulsive nature of the $\sigma^{*}$ anion's energy surface) to form an $\mathrm{R}-\mathrm{S}$ radical and an $-\mathrm{S}-$ $\mathrm{R}^{\prime}$ anion. The yield of bond cleavage is governed by competition between fragmentation on the $\sigma^{*}$ surface and autodetachment. The ab initio calculations of ref 1 were carried out on a very simple model of the disulfide shown in Figure 1, the $\mathrm{H}_{3} \mathrm{C}-\mathrm{S}-$ $\mathrm{S}-\mathrm{CH}_{3}$ molecule. The $\mathrm{R}-\mathrm{S}-\mathrm{S}-\mathrm{R}^{\prime}$ neutral and corresponding anion potential energy curves for dimethyl disulfide as functions of the $\mathrm{S}-\mathrm{S}$ distance are depicted below in Figure 2.

Although there had been previously very good theoretical studies $^{3}$ of reductive $S-S$ bond cleavage, they have not focused on the regions of the anion's energy surface, at which this species is electronically metastable to autodetachment. For

\footnotetext{
* Corresponding author. E-mail: simons@chemistry.utah.edu.

A holder of a Foundation for Polish Science (FNP) Award.

$\doteqdot$ University of Utah.

$\S$ University of Gdansk.
}

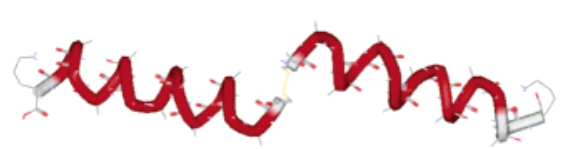

Figure 1. Structure of an $\left(\mathrm{AcCA}_{15} \mathrm{~K}+\mathrm{H}\right)_{2}{ }^{2+}$ disulfide-linked dimer from ref 2 . The disulfide linkage is at the center, and the two protonated sites are at the left and right ends.

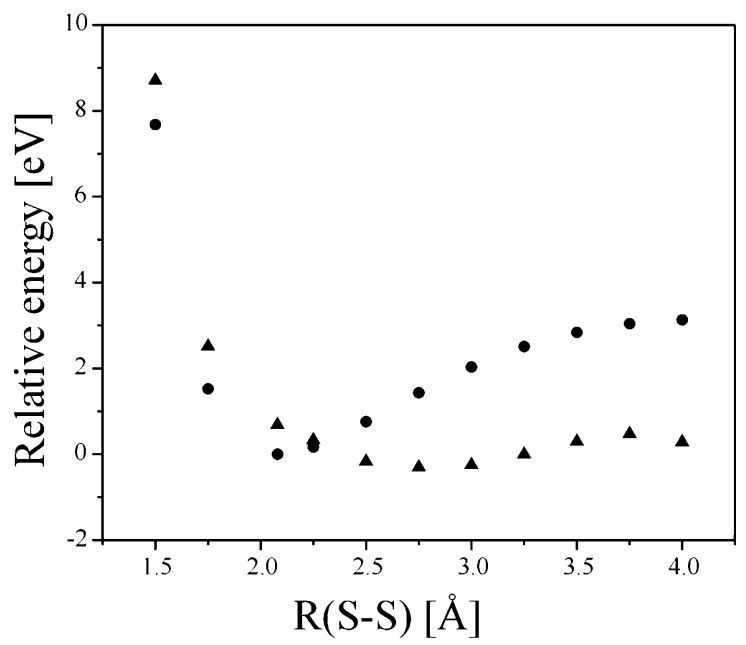

Figure 2. Energies of the dimethyl disulfide neutral (circles) and $\sigma^{*}$ anion (triangles) as functions of the $\mathrm{S}-\mathrm{S}$ bond length $(\AA)$ with all other geometrical degrees of freedom relaxed to minimize the energy.

example, they did not consider direct near-vertical electron attachment to the $\sigma^{*}$ orbital of the $\mathrm{S}-\mathrm{S}$ bond to produce an unstable $\sigma^{*}$ anion. Rather, these studies were limited to treating 


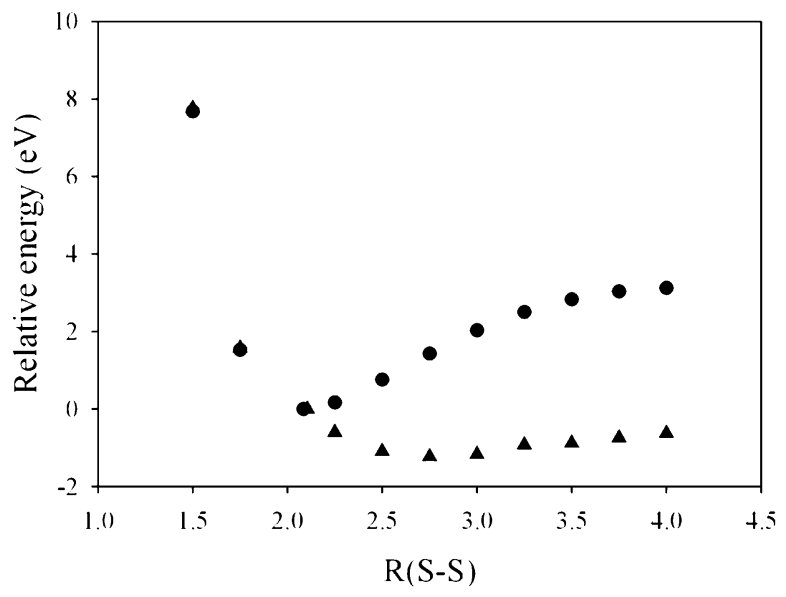

Figure 3. Neutral (dots) and $\sigma^{*}$ anion (triangles) curves of MeSSMe in the presence of two +1 charges $30 \AA$ from the midpoint of the $\mathrm{S}-\mathrm{S}$ bond.

the anion at geometries where it is electronically bound as, for example, in the $\mathrm{R}-\mathrm{S}^{\bullet} . . . \mathrm{SR}^{\prime}$ radical-anion complex. Nevertheless, the papers cited in ref 3 contributed much to clarifying a mechanism by which $\mathrm{S}-\mathrm{S}$ bonds can be reductively cleaved in biological molecules.

To return to what our earlier study of $\mathrm{S}-\mathrm{S}$ bond rupture showed, Figure 2 suggests that the near-vertical attachment of an electron into the $\mathrm{S}-\mathrm{S} \sigma^{*}$ orbital of $\mathrm{MeS}-\mathrm{SMe}$ would require an electron with kinetic energy of ca. $0.9 \mathrm{eV}$ and would generate the $\sigma^{*}$ anion on a reasonably repulsive (and electronically metastable) part of its energy surface. This results in the wellknown dissociative electron attachment (DEA) process ${ }^{4}$ that has been well-studied experimentally for $\mathrm{MeS}-\mathrm{SMe}$. We refer to this specific DEA event as "direct" because the electron enters an antibonding orbital of the same bond that subsequently fragments. Figure 2 also suggests that lower-energy electrons (e.g., even zero-energy electrons) can attach to the $\sigma^{*} \mathrm{~S}-\mathrm{S}$ orbital, but only if the $\mathrm{S}-\mathrm{S}$ bond is stretched to near $2.25 \AA$, which would require ca. $0.5 \mathrm{eV}$ of vibrational excitation. Of course, except at considerably elevated temperatures, such high vibrational excitation is extremely improbable. However, the Heisenberg widths (an autodetachment rate of $10^{14} \mathrm{~s}^{-1}$ corresponds to a width of $0.4 \mathrm{eV}$ ) of such metastable states can be substantial, and they vary from zero, where the anion and neutral curves cross, to larger values at shorter bond lenths. This then suggests that electron attachment can be expected at lower electron energies (due to the Heisenberg width of the $\sigma^{*}$ anion) than predicted from the near-vertical attachment energy of ca. $0.9 \mathrm{eV}$. Throughout this manuscript, it is important to keep in mind the fact that the $\sigma^{*}$ and $\pi^{*}$ resonance states we consider will have widths ranging from a few tenths of an $\mathrm{eV}$ to more than $3 \mathrm{eV}$ with the widths being small near the anion-neutral curve crossing and larger at smaller bond lengths where the anion lies further above the energy of the neutral.

The primary focus in ref 1 was to consider the effects that proximal positively charged groups can have on the DEA process. Specifically, we considered the Coulomb stabilization that one or more nearby positive groups (e.g., the protonated Lys sites in the molecule shown in Figure 1) can have on the nascent $\sigma^{*}$ anion. As an example of the effects of Coulomb interactions, in Figure 3 we show the MeS-SMe neutral and $\mathrm{MeS}-\mathrm{SMe}^{-} \sigma^{*}$ anion potentials, as in Figure 2, but calculated in the presence of two +1 charges, each $30 \AA$ from the midpoint of the $\mathrm{S}-\mathrm{S}$ bond (i.e., one +1 charge on one side of the bond and the other +1 charge on the other side, both lying along the $\mathrm{S}-\mathrm{S}$ bond direction). Clearly, in comparison with Figure 2, the

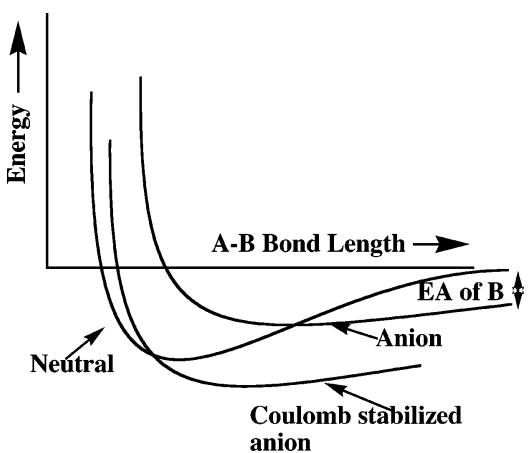

Figure 4. Prototypical $\mathrm{A}-\mathrm{B}$ neutral and $\mathrm{A}+\mathrm{B}^{-} \sigma^{*}$ anion curves as well as the Coulomb-stabilized anion curve. The EA of the $\mathrm{B}$ radical is also noted.

$\sigma^{*}$ anion curve in Figure 3 is lower in energy by a substantial amount relative to the energy of the neutral. This causes the anion curve to intersect the neutral at smaller $\mathrm{S}-\mathrm{S}$ separations (e.g., at bond lengths that may be accessed in the zero-point vibration of the $S-S$ bond) and at much lower energy.

It turns out that the energy-lowering of the $\sigma^{*}$ anion curve can be estimated in terms of the Coulomb potential produced by the two +1 charges. For example, when the two charges are $30 \AA$ distant, the Coulomb energy at the midpoint of the $\mathrm{S}-\mathrm{S}$ bond is $2(14.4 \mathrm{eV} \AA) / 30(\AA)=0.96 \mathrm{eV}$; when the two charges are only $10 \AA$ away, the Coulomb stabilization energy is 2.88 $\mathrm{eV}$. The (assumed) rigidity of the compounds shown in Figure 1 caused by their helical subunits allowed us to know the distances between the $\mathrm{S}-\mathrm{S}$ bond and the two +1 sites, so these species provided excellent support for postulation that our model $\mathrm{MeS}-\mathrm{SMe}$ compounds were designed to probe.

On the basis of the results of such studies, we suggested in ref 1 that Coulomb potentials produced by nearby positive charges could stabilize the $\sigma^{*}$ metastable anion states to an extent that might render them electronically stable. Under such circumstances, the endothermic DEA process illustrated in Figure 2 might be made exothermic or thermoneutral and thus able to effect bond breakage at a much higher yield.

Let us now consider in more generality the role of Coulomb stabilization and the impact of the intrinsic electron binding energy of the species formed when bond cleavage occurs. In Figure 4, we show prototypical $\mathrm{A}-\mathrm{B}$ neutral and $\mathrm{A}+\mathrm{B}^{-}$anion curves that result in such direct DEA studies. The key observations to make are as follows:

1. The energy at the intersection of the $\sigma^{*}$ anion and neutral curves depends on the electron affinity (EA) of the fragment B formed when the $\mathrm{A}-\mathrm{B}$ bond is broken. The larger the EA of $B$, the lower on the neutral's energy profile and the shorter the $\mathrm{A}-\mathrm{B}$ distance the intersection will occur. This thermodynamic driving force is essential to keep in mind when considering which bonds within a large polyatomic species will be most susceptible to DEA at low electron energies (i.e., bonds that form radicals having large EAs are good candidates). Of course, the radial "sizes" of the A and B fragments, which govern their shorter-range repulsion, will also contribute to determining where this crossing occurs.

2. The $\sigma^{*}$ anion curve in the presence of stabilizing ${ }^{5}$ Coulomb potentials is more or less uniformly lowered (i.e., at all $R$ values) in energy by an amount equal to the Coulomb energy.

We have seen such behavior in all of our ab initio studies ${ }^{1}$ to date. This means that the bonds closest to the sources of the Coulomb potentials will be most affected and thus most likely candidates for Coulomb-assisted DEA. In a fairly rigid compound such as that shown in Figure 1, the distances to the 


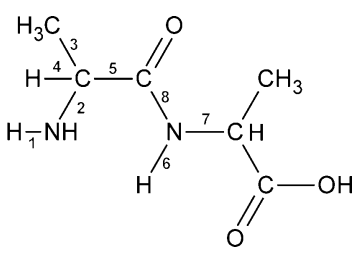

Figure 5. Prototype molecule (Ala-Ala) used in this study with the $\sigma$ bonds whose cleavage we studied numbered $1-8$.

positive sites are reasonably fixed. In a compound containing, for example, a protonated amine site that is attached to a flexible side chain, the distance to the positive site will fluctuate as the molecule moves. In such a case, one must imagine a $\sigma^{*}$ anion curve whose energy (relative to that of the neutral) is fluctuating but which may, at certain times, intersect the neutral curve at a low energy.

Our work in this area is an effort to help interpret the mechanism of bond cleavage arising in gas-phase mass spectrometric experiments on charged peptides and proteins. In particular, the use of electron capture dissociation (ECD) methods ${ }^{6}$ for fragmenting such biomolecules gives rise to very specific bond cleavage patterns, but the mechanisms involved are not yet fully understood. To extend our study to species that contain a range of bonds characteristic of many peptide samples studied by this kind of experimental techniques, we decided to examine the neutral and anion energy surfaces associated with cleaving each of the numbered bonds in the prototype molecule (Ala-Ala dipeptide) shown in Figure 5.

In each component of the present study, we consider attaching an electron to the $\sigma^{*}$ orbital of one of the eight bonds labeled in Figure 5. For each such attachment, we need to determine, using the special techniques detailed in the following section, where the $\sigma^{*}$ anion state lies energetically relative to the neutral molecule at geometries characteristic of the neutral. Of necessity, this requires us to calculate the energy of the anion at geometries where it is electronically metastable. We emphasize that, in such cases, the energy we obtain gives us an approximation to the so-called position (i.e., the center of the Heisenberg broadened anion state) of the resonance state. However, we need to keep in mind that this state's energy is not "sharp" but has a width to it.

Nevertheless, these data allow us to estimate the kinetic energy an electron must possess to attach to this $\sigma^{*}$ orbital at various bond lengths; these estimates will be more specific near the anion-neutral curve crossing where the anion is not so broadened and less so at shorter bond lengths. We do likewise for the carbonyl $\mathrm{C}=\mathrm{O} \pi^{*}$ orbital involving the carbon atom involved in bond 8 . We then explore how the energy of the nascent anion formed by such an electron attachment varies as the bonds shown in Figure 5 are stretched. These same steps are repeated with the molecule in the presence of Coulomb potentials at various positions designed to simulate, for example, the presence of protonated amine sites. By this procedure, we are able to gain insight into which bonds will be most energetically amenable to dissociative electron attachment, both in the absence of any stabilizing positive charges and with stabilizing Coulomb potentials present.

\section{Methods}

Prior to stretching any of the eight bonds, we optimized the geometry of the neutral molecule shown in Figure 5 at the Hartree-Fock level using aug-cc-pVDZ basis sets ${ }^{7}$ and calculated all vibrational frequencies to make sure the structure thus found was indeed a minimum on the energy surface. All of our calculations were carried out using the Gaussian 03 suite $^{8}$ of codes.

The size of the molecule, combined with the extremely large number of geometries at which we needed to evaluate the energies and the fact that special techniques involving many calculations per geometry were needed (see later) to deal with the metastable anions, limited our treatment to the uncorrelated unrestricted Hartree-Fock (UHF) level. We know from our earlier work on $\mathrm{S}-\mathrm{S}$ bond cleavage, which was carried out using ab initio methods including electron correlation, that the UHF treatment of the anion will tend to underestimate its stability. This, in turn, means the energies at which anion-neutral curve crossings occur and vertical attachment energies are calculated using the UHF wave function will be too high (probably by a few to several tenths of an $\mathrm{eV}$ ). However, because the $\sigma^{*}$ and $\pi^{*}$ anion states we are considering have large Heisenberg widths (ca. $0.3-3 \mathrm{eV}$ ) at some of the bond lengths we need to examine, we believe it is a reasonable compromise to use the UHF method. Of course, this limits the absolute accuracy of the critical energies we extract from our calculations, but in this work, we are only trying to gain a qualitative picture of which bonds are most susceptible to Coulomb-assisted electron attachment and which are too strong to be cleaved in this process. For the one bond that we found (see later) to be most likely susceptible to cleavage by electron attachment, we subsequently repeated our UHF-level study at the second-order MøllerPlesset (MP2) correlated level of theory to gain a more accurate estimate of the relative energies of the neutral and anion states.

To generate the neutral molecule and anion energies as functions of each of the bond lengths ( $R$ 's) shown in Figure 5, we performed such UHF calculations at a range of $R$-values but with all other geometrical degrees of freedom frozen at the values they have in the minimum-energy structure. As such, the anion energies we obtain are best viewed as appropriate to the vertical attachment of an electron and, because of our use of the UHF method, probably overestimate the anion-neutral energy splittings.

As mentioned already, the anion calculations are especially problematic at $R$-values where the anion's energy lies above that of the neutral (i.e., when the anion is metastable). In such cases, great care must be taken to avoid having the anion's wave function undergo variational collapse (i.e., to describe an electron distant from the neutral molecule and having little kinetic energy). For example, when stretching the bond labeled 6 in Figure 5, we had to be careful to monitor the anion's orbital occupancy to guarantee that the $\mathrm{N}-\mathrm{H} \sigma^{*}$ orbital was indeed the singly occupied molecular orbital. At large $R$-values, this is rather straightforward, because at such distances, the anion is electronically stable. However, at $R$-values where the $\mathrm{N}-\mathrm{H} \sigma^{*}$ anion's energy lies above that of the neutral molecule, we had to employ a special technique that we now describe for such an electronically metastable state.

In the method we used to overcome these problems for metastable anions, we increased the nuclear charges on the two atoms (e.g., $\mathrm{N}$ and $\mathrm{H}$, when stretching the $\mathrm{N}-\mathrm{H}$ bond) involved in the bond cleavage by an amount $\delta q$ and carried out the anion calculation. These increased nuclear charges cause the valence components of the $\mathrm{N}-\mathrm{H} \sigma^{*}$ anion state to be differentially lowered in energy and to thus cause this state to become electronically stable relative to the neutral molecule. In this way, we were able to make sure that the anion energy and orbital occupancy we obtained in our UHF calculations corresponded to the proper $\sigma^{*}$ anion. By employing this $\delta q$ charge increase 


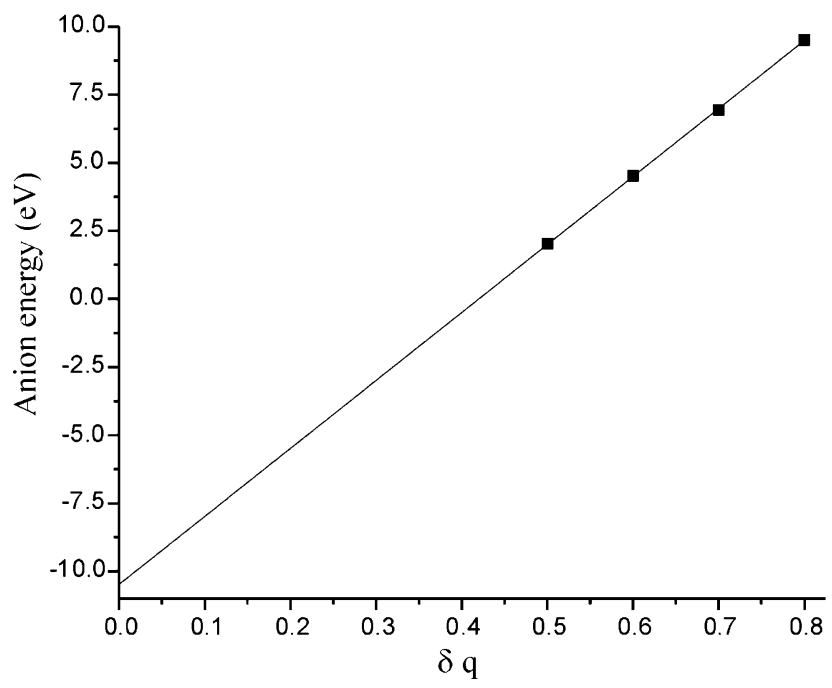

Figure 6. Plot of the energy of the anion relative to that of the neutral in which an electron is in a $\mathrm{N}-\mathrm{H} \sigma^{*}$ orbital vs the stabilizing partial charge $\delta q$ added to the $\mathrm{N}$ and $\mathrm{H}$ atoms.

technique for a range of $\delta q$ values (e.g., $\delta q=0.05,0.1$, through 1.0 was typical) within which the $\sigma^{*}$ anion is electronically stable, we could then plot the stable energies $E(\delta q)$ of the $\sigma^{*}$ anion versus $\delta q$ and extrapolate to $\delta q=0$ to evaluate the metastable $\sigma^{*}$ anion's energy. An example of such an extrapolation is shown in Figure 6. We have used this method with considerable success in other studies ${ }^{1,9}$ of metastable anions, so we know it to be reliable.

As a result of such a treatment, we were able to compute the electronic energy of the neutral molecule as a function of the bond lengths shown in Figure 5 as well as the vertical electronic energy (actually, an estimate of the position or middle of the Heisenberg broadened energy distribution) of the anion state of interest. In particular, we were able to evaluate the anion's energy both at geometries where it is electronically stable and where it is metastable, the latter being especially important for estimating the vertical electron attachment energies that relate to DEA processes.

In addition to carrying out such calculations in the absence of any stabilizing Coulomb potentials, we repeated each such calculation with Coulomb potentials present. In all such calculations, we placed two +1 charges at 10 or $30 \AA$ from the two atoms involved in the bond being cleaved and in directions lying along the bond and repeated the evaluation of the neutral and (vertical) anion energies. Such positive charges generate 2(14.4/ $10)=2.88 \mathrm{eV}$ and $2(14.4) / 30=0.96 \mathrm{eV}$ of stabilization at the bond midpoints, respectively. These positive charges could represent, for example, the totality of positive charges that may arise in a multiply protonated gas-phase sample of a peptide or protein.

Because we find that the presence of $a+1$ charge stabilizes the anion curves in a highly predictable manner (i.e., by lowering the $\sigma^{*}$ curve by $14.4 \mathrm{eV} / R$, where $R$ is the distance in angstroms to the +1 charge), we can predict the energy and shape of the $\sigma^{*}$ anion curve for arbitrary values of the local Coulomb potential. This, in turn, allows us to utilize our findings to predict the effects, for example, of a protonated amine site on a side chain whose distance to a given bond may even fluctuate as the molecule undergoes thermal motions. So, even though we carry out calculations for only certain strengths of the Coulomb potential, we suggest that our data can be used to predict under what Coulomb potentials a given bond will or will not be susceptable to exothermic DEA.

\section{Results}

A. Direct Attachment to $\sigma^{*}$ Orbitals. First, let us consider cases in which the incident electron attaches directly into a $\sigma^{*}$ orbital of each of the bonds labeled in Figure 5. On the basis of our discussion surrounding Figure 4, we should expect that cleavage of none of these bonds should, in the absence of Coulomb stabilization, produce $\sigma^{*}$ anion curves that intersect the corresponding neutral curves at low energy and short distances. Why? Because the $\mathrm{H}^{\bullet}, \mathrm{R}_{3} \mathrm{C}^{\bullet}, \mathrm{R}_{2} \mathrm{~N}^{\bullet}$, and $[\mathrm{RC}=\mathrm{O}]^{\bullet}$ radicals formed when these bonds are cleaved do not have large electron affinities. $\mathrm{H}^{\bullet}$ and $\mathrm{R}_{2} \mathrm{~N}^{\bullet}$ have EAs in the $0.5-0.7 \mathrm{eV}$ range and $\mathrm{R}_{3} \mathrm{C}^{\bullet}$ has an even smaller $\mathrm{EA}(<0.1 \mathrm{eV})$. In contrast, the $\mathrm{R}-\mathrm{S}^{\bullet}$ radical formed upon breaking the $\mathrm{S}-\mathrm{S}$ bond in the species shown in Figure 1 has an EA near $1.8 \mathrm{eV}$. Hence, the anion curves arising when bonds $1-8$ are broken will not lie much below the neutral at large $R$ values, and thus, the repulsive anion curve will intersect the neutral at large $R$ values and high energy.

In Figure 7, we show the neutral and anion curves associated with cleaving each of the bonds shown in Figure 5. In these figures, we show the anion curve when no stabilizing +1 charges are present and when two +1 charges are located $10 \AA$ from the atoms in the bond being cleaved and lying along this bond direction. In the sixth of these figures, we also show anion curves when two +1 charges are 20 or $30 \AA$ from the bond's atoms. The reason we do not show the 20 and $30 \AA$ data for all of the cases is that we verified that the anion energy $E(X)$ in the presence of two charges $X \AA$ away from the bond is essentially identical to the anion energy $E^{\circ}$ in the absence of any charges minus the Coulomb energy computed as $14.4 \mathrm{eV} \AA / X(\AA): E(X)$ $=E^{\circ}-14.4 / X$. Finally, in the last of the figures, we show the effects of the +1 charges' Coulomb potential on the neutral curve for stretching the bond labeled 3 (the dependences on $X$ for stretching bonds $1-8$ are very similar). Not surprisingly, the energy of the neutral species is much less affected than that of the $\sigma^{*}$ anion, regardless of which of the eight bonds are stretched. For this reason, it is reasonably accurate in estimating the vertical electron attachment energies to examine where the anion curves in the absence and in the presence of the two +1 charges intersect the neutral curve calculated with no +1 charges present.

Before discussing the significance of these data, we wish to point out that, for the particularly strong $\sigma$ bonds whose cleavages are treated here, there is little reliable experimental DEA data. The absence of good data is related to the fact that, for strong $\sigma$ bonds whose cleavage does not lead to radicals having large EAs, the $\sigma^{*}$ anion curve lies high above the neutral curve near the equilibrium bond length of the netural. Therefore, the $\sigma^{*}$ anion state has a high energy and a correspondingly very short lifetime (ca. $10^{-14} \mathrm{~s}$ or shorter). The short lifetime produces a very broad Heisenberg width and thus makes the determination of the center of the DEA cross-section difficult to determine. Moreover, the short lifetime causes detachment to overwhelm dissociation of the $\sigma^{*}$ anion, thus making the bond-cleavage yield of the DEA process very low. All of these issues conspire to make DEA data on the bond we are studying very scarce. For these reasons, we are not in a position to offer much laboratory evidence to support the anion energy curves shown in Figure 7. However, we note that our earlier work on $\mathrm{S}-\mathrm{S}$ bond cleavage ${ }^{1}$ was not plagued by the lack of experimental evidence, and the latter turned out to be very much in agreement with our findings, thus providing support for our approach to the problem at hand. 
1) $\mathrm{N}-\mathrm{H}$ bond

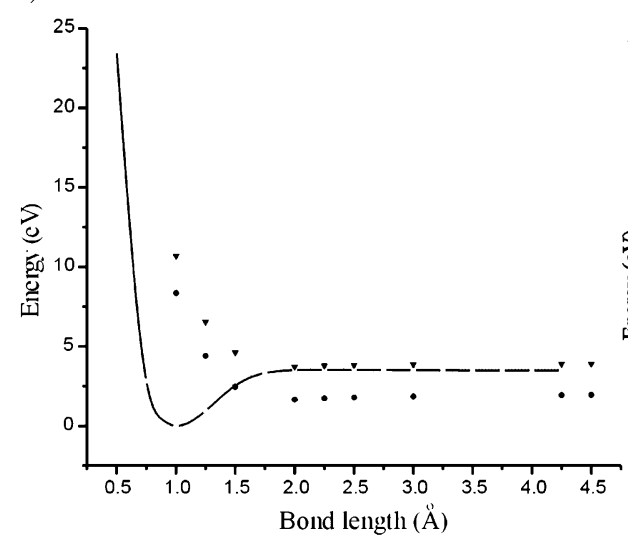

2) $\mathrm{N}$ - C bond

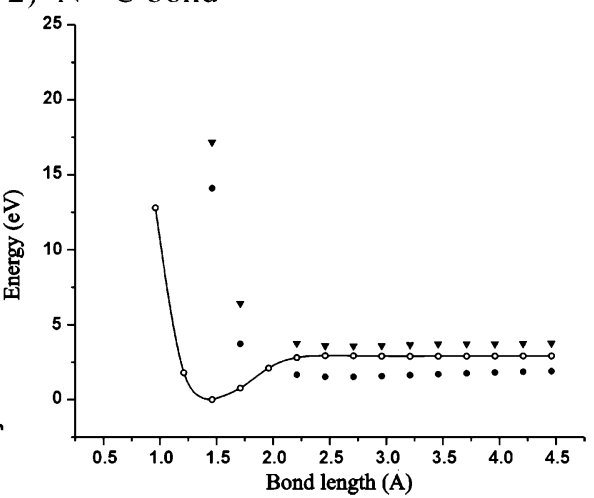

3) C - C $\left(\mathrm{sp}^{3}\right)$ bond

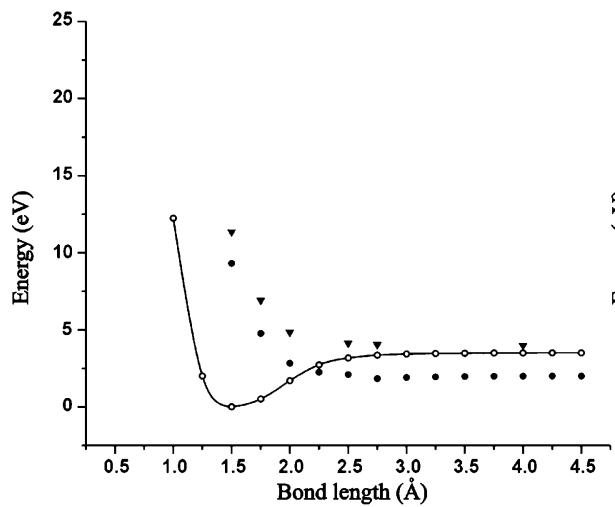

5) $\mathrm{C}-\mathrm{C}\left(\mathrm{sp}^{2}\right)$ bond

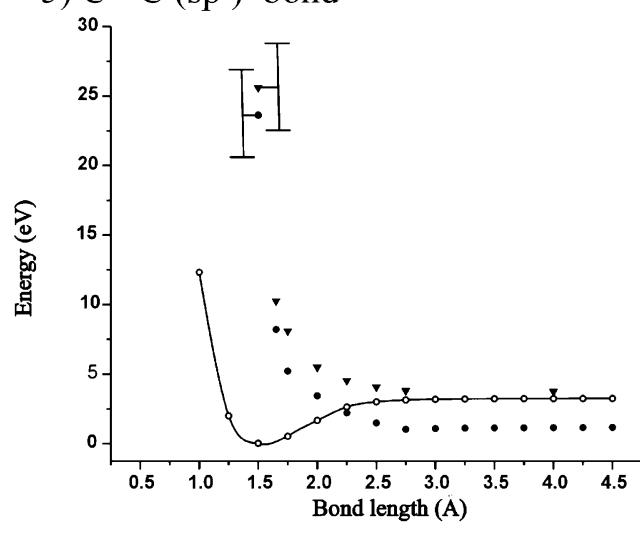

4) $\mathrm{C}-\mathrm{H}$ bond

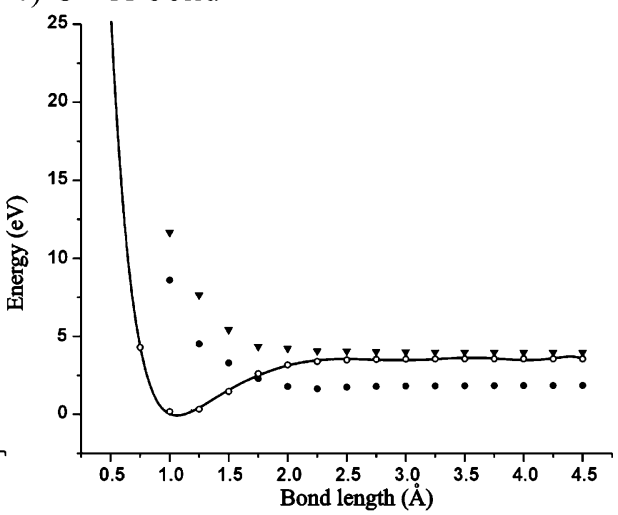

6) $\mathrm{N}-\mathrm{H}$ bond

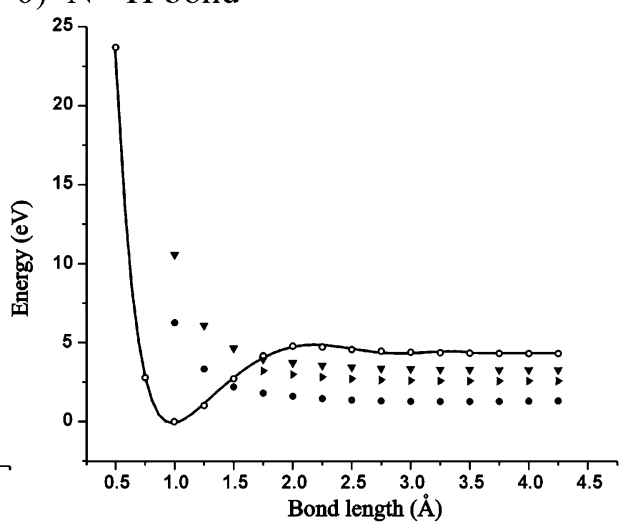

7) $\mathrm{N}-\mathrm{C}$ bond

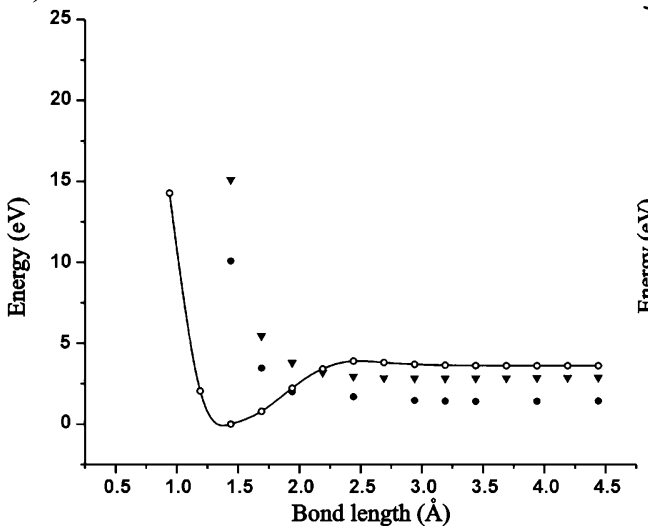

3) $\mathrm{C}-\mathrm{C}\left(\mathrm{sp}^{3}\right)$ bond

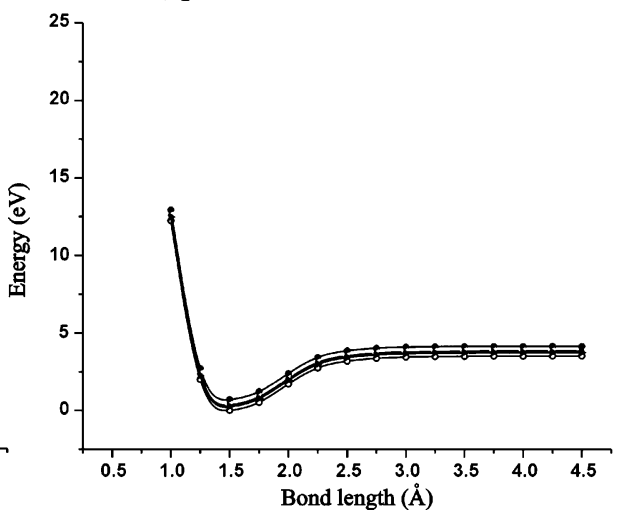

Figure 7. Energies of the neutral (open circles) and $\sigma^{*}$ anion with no +1 charges (triangles) and with two +1 charges $10 \AA$ from the bond's atoms (filled circles). In the sixth figure, the anion curves are also shown when the two +1 charges are 20 or $30 \AA$ distant, and in the last figure, the neutral energy is shown for no charges and for two +1 charges 10,20 , and $30 \AA$ distant and bond 3 is stretched. 
Now, let us turn to make a few observations about the data shown in Figure 7:

1. For the situations in which a $\mathrm{C}-\mathrm{C}$ bond is cleaved, forming two carbon radicals, the corresponding carbon-centered anion should have a very small $(<0.1 \mathrm{eV})$ positive electron binding energy. In our graphs, the anion curves corresponding to these cases do not display this small stability, because the anion energies we plot are computed as vertical attachment energies and at the uncorrelated UHF level. More accurate representations can be achieved by simply shifting the $\sigma^{*}$ anion curves downward to achieve an EA of ca. $0.03 \mathrm{eV}$ at large $R$ values (i.e., to reproduce the EA of such carbon radicals).

2. For all of the bonds whose cleavage is represented by these data, the vertical attachment of an electron to the $\sigma^{*}$ orbital of that bond is highly $(>6 \mathrm{eV})$ endothermic in the absence of Coulomb stabilization. Even if one were to shift the anion curves downward to achieve more correct large $R$ energies (i.e., to reproduce the correct correlated EAs of the fragments), the vertical attachment energies remain greater than $5 \mathrm{eV}$. Even taking into consideration the facts discussed earlier that the UHF method likely places the anion too high (by a few tenths of an $\mathrm{eV}$ ) and that the anion states are Heisenberg broadened, the data of Figure 7 suggest that near-vertical attachment into a $\sigma^{*}$ orbital is quite endothermic.

3. Even when Coulomb stabilization of $2.88 \mathrm{eV}$ is present (i.e., equivalent to two +1 charges $10 \AA$ distant or one +1 charge $5 \AA$ away), the $\sigma^{*}$ anion curve vertically lies far (>3 $\mathrm{eV}$ ) above the neutral and intersects the neutral at large $R$ values and high $(>2 \mathrm{eV})$ above the neutral's minimum. Again, even if one were to shift the anion curves downward to achieve more correct large $R$ energies, these conclusions would change little.

It therefore seems unlikely that such strong $\sigma$ bonds will be susceptible to cleavage by a direct low-energy DEA mechanism even when positive charges are nearby. To effect vertical attachment to such a $\sigma$ bond lying more than $6 \mathrm{eV}$ above the neutral, one would have to have, for example, a protonated amine site within $2.4 \AA$. Of course, one can imagine multiply protonated species for which the total Coulomb stabilization at a $\sigma$ bond site could move that site's $\sigma^{*}$ anion below the corresponding neutral, but such cases are probably not common. The species shown in Figure 1 represents such an unusual case in which two +1 charges as distant as $30 \AA$ are able to allow direct DEA to occur. It is the weakness of the $\mathrm{S}-\mathrm{S}$ bond (which causes the energy of the $\sigma^{*}$ orbital to be low-lying) and the magnitude (ca. $1.8 \mathrm{eV}$ ) of the EA of the $\mathrm{S}-\mathrm{R}^{\prime}$ radical formed upon bond cleavage that make this case special.

For the very strong $\mathrm{C}-\mathrm{C}, \mathrm{C}-\mathrm{H}, \mathrm{C}-\mathrm{N}$, and $\mathrm{N}-\mathrm{H} \sigma$ bonds whose data are summarized in Figure 7 , the bond strengths and radical EAs do not combine to permit efficient direct DEA when low-energy (i.e., $<3 \mathrm{eV}$ ) electrons are involved. We therefore expect that Coulomb-assisted DEA will not be very effective in gaseous peptide samples except for bonds that happen to be very near two or more protonation sites (i.e., for which the total Coulomb stabilization approaches or exceeds $5 \mathrm{eV}$ ). This negative finding is actually good news, because it suggests that the fragmentation patterns involved in ECD cleavage of gaseous positively charged peptide samples will be less complicated than one might expect if it were probable for such strong $\sigma$ bonds to be ruptured.

B. Attachment to $\pi^{*}$ Orbitals Followed by Rupture of Nearby $\boldsymbol{\sigma}$ Bonds. Now, let us consider an alternative mechanism by which an electron can attach to the molecule shown in Figure 5 and effect a $\sigma$ bond cleavage. In particular, we consider what

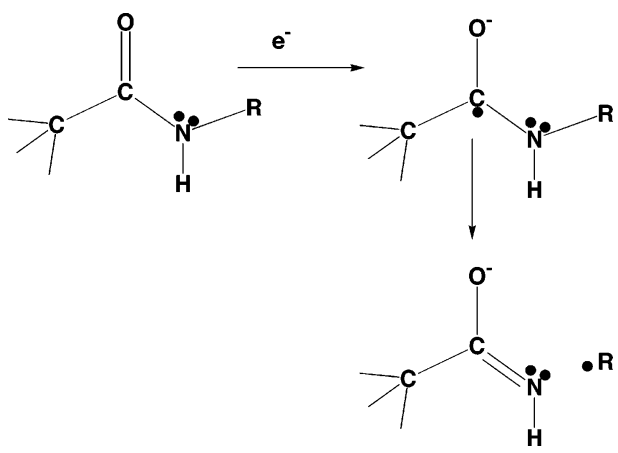

Figure 8. Idealized mechanism involving electron capture into a $\mathrm{C}=$ $\mathrm{O} \pi^{*}$ orbital and subsequent rupture of a $\mathrm{N}-\mathrm{R}$ bond combined with formation of a new $\mathrm{C}-\mathrm{N} \pi$ bond.

happens when an electron enters one of the carbonyl group $\pi^{*}$ orbitals. Clearly, these orbitals are among the lowest-energy vacant orbitals of this prototype molecule, so it makes sense to consider them when examining low-energy electron-induced fragmentation. Moreover, the autodetachment lifetimes of such $\pi^{*}$ states tend to be longer than those of the $\sigma^{*}$ states we just analyzed. Hence the bond-cleavage yields can be larger when attachment occurs at a $\pi^{*}$ orbital. However, the process in which an electron enters a $\mathrm{C}=\mathrm{O} \pi^{*}$ orbital yet generates a $\sigma$ bond cleavage involving some other pair of atoms ${ }^{10}$ is a bit more complicated that the direct attachment to a $\sigma^{*}$ orbital that we treated earlier.

Let's consider a specific example to illustrate the added complexity: cleavage of the bond labeled 7 in Figure 5. One can imagine a mechanism (see Figure 8) in which an electron enters the $\mathrm{C}=\mathrm{O} \pi^{*}$ orbital, thus rupturing the $\pi$ bond to generate an $-\mathrm{O}^{-}$anionic site that is adjacent to a carbon radical. After this, if the bond connecting the nitrogen to the $\mathrm{R}$ group (i.e., the $\mathrm{N}-\mathrm{C}_{\alpha}$ bond) were stretched, this bond could break while allowing a new $\mathrm{C}-\mathrm{N} \pi$ bond to be formed to partially offset the energy cost involved in the $\mathrm{N}-\mathrm{C}_{\alpha}$ bond cleavage.

As a matter of fact, the bond-reforming process outlined in Figure 8 results in the fragmentation pattern ${ }^{11}$ that is very characteristic in low-energy ECD experiments on positively charged gaseous peptide samples (in which the fragments are labeled $\mathrm{c}$ and $\mathrm{z}$ ), so it is critical to this study that we examine its energy profile. Alternative means of energizing the sample (e.g., collisional, electron impact, or infrared multiphoton absorption) tend to cause fragmentation ${ }^{12}$ at the carbonyl carbon $\mathrm{C}-\mathrm{N}$ bond producing fragments labeled $\mathrm{b}$ and $\mathrm{y}$.

Let's consider cleaving this $\mathrm{C}-\mathrm{N} \sigma$ bond connecting the carbonyl carbon to the nitrogen instead of the $\mathrm{N}-\mathrm{C}_{\alpha} \sigma$ bond. This is a more energy-intensive process for the ECD mechanism because it generates an -NHR anion (which has an electron binding energy of ca. $0.6 \mathrm{eV}$ ) and a $\mathrm{R}_{3} \mathrm{C}-\mathrm{C}=\mathrm{O}^{\bullet}$ radical; the pathway shown in Figure 8 forms considerably more stable species because of the larger EA of the $-\mathrm{O}$ site and the formation of the $\mathrm{C}=\mathrm{N} \pi$ bond. Nevertheless, in this study, we did indeed examine cleavage of the $\mathrm{C}-\mathrm{N} \sigma$ bond to determine its energy profile as well (see following text).

What makes the DEA process involving attaching an electron to a $\pi^{*}$ orbital more complicated to study is that there are two or more anion states that must be considered. In contrast, the direct $\sigma$ bond cleavage events treated earlier involve only the energy curve of the parent neutral and that of the dissociative $\sigma^{*}$ anion. In Figure 9, we illustrate these differences.

In the top of Figure 9, we show the bound potential energy curve of the neutral species as a function of the length of the bond that is broken as well as the repulsive curve of the anion 

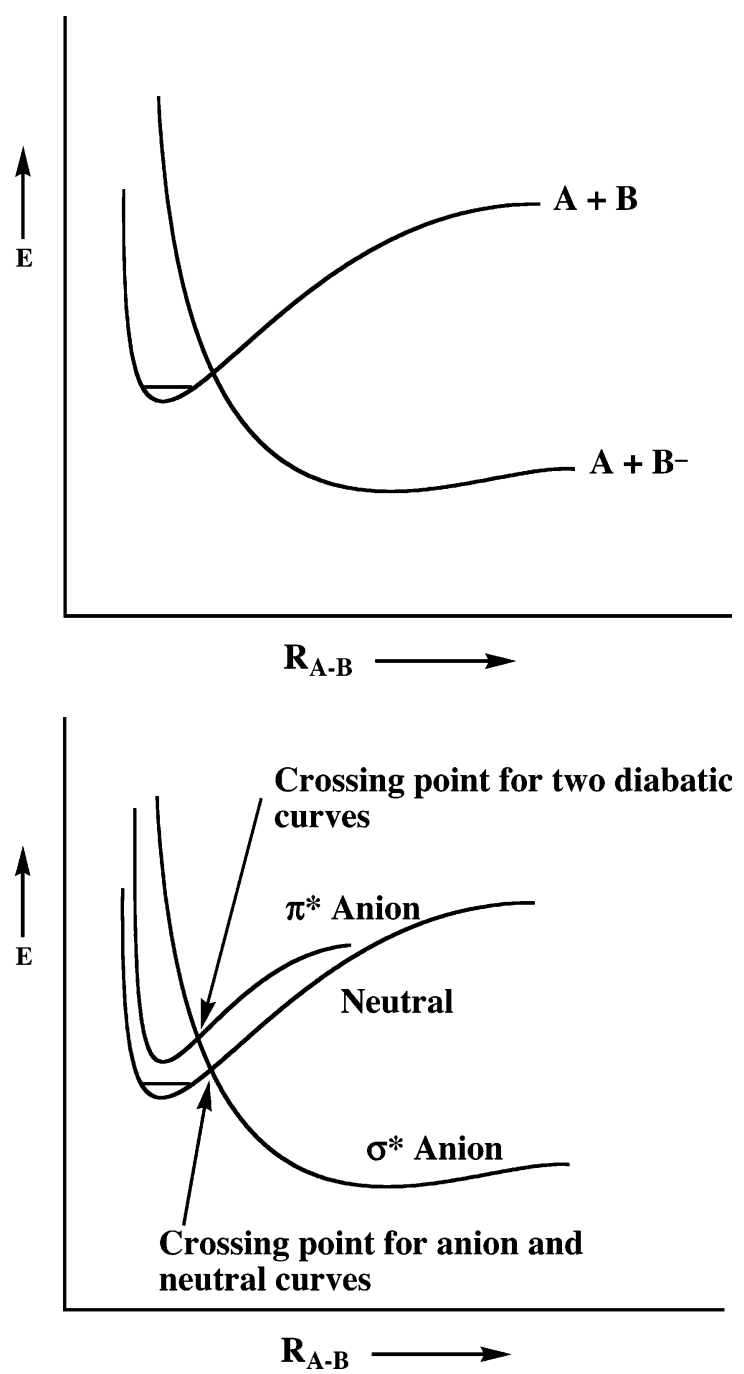

Figure 9. Neutral and $\sigma^{*}$ anion curves associated with direct DEA (top) and neutral, $\pi^{*}$, and $\sigma^{*}$ anion curves associated with indirect DEA (bottom).

formed when an electron attaches directly to the $\sigma^{*}$ orbital of that bond. In the bottom of Figure 9, we show the energy curves of the bound neutral, of the repulsive $\sigma^{*}$ anion, and of the anion formed when the electron enters a $\pi^{*}$ orbital (e.g., of the $\mathrm{C}=\mathrm{O}$ group) near the bond that is ultimately broken.

In the indirect DEA case, bond cleavage can be viewed as involving two steps:

i. The electron attaches to the $\pi^{*}$ orbital forming an anion whose potential curve has a minimum rather than being purely repulsive. This barrier on the $\pi^{*}$ curve suggests that rupture of the $\sigma$ bond will not be prompt as in the direct DEA process in which the repulsive $\sigma^{*}$ anion's curve is immediately accessed.

ii. The $\pi^{*}$ anion undergoes electronic coupling with the $\sigma^{*}$ state to produce an adiabatic surface having a barrier (near the crossing point indicated in Figure 9) that must be surmounted or tunneled through. ${ }^{13}$ This coupling is how the $\pi^{*}$ anion eventually allows bond cleavage to occur by forming the dissociative $\sigma^{*}$ anion.

Of course, as in the direct DEA process, when the anion exists on the adiabatic surface to the left of the $R$ value where this anion curve crosses the neutral, the anion is subject to electron autodetachment (at a rate of ca. $10^{14} \mathrm{~s}^{-1}$ ). The yield of bond fragmentation is determined by the competition between detachment and movement over (or tunneling through) the barrier on the adiabatic surface.
Keeping this background in mind, let us now examine the potential curves we obtain when an electron is placed into the central carbonyl's $\mathrm{C}=\mathrm{O} \pi^{*}$ orbital while $\sigma$ bonds 5 , 7, and 8 near the carbonyl group are stretched (see Figure 10).

What do these potential curves tell us? First, it is useful to note that a near-vertical attachment of an electron to the $\mathrm{C}=\mathrm{O}$ $\pi^{*}$ orbital is predicted to require an electron having ca. $2.5-$ $2.8 \mathrm{eV}$ of kinetic energy which is considerably lower than the $>6 \mathrm{eV}$ needed for the $\sigma$-bond attachments detailed earlier. Of course, as we noted earlier, all of these energies are probably a bit high because we used the noncorrelated UHF method, but there is no doubt that the $\pi^{*}$ attachment is less endothermic than the $\sigma^{*}$ attachment.

The data in Figure 10 refer to the situation in which no stabilizing +1 charges are present. In the presence of two +1 charges within $10 \AA$ (or the equivalent Coulomb potential from other sources), the $\pi^{*}$ and $\sigma^{*}$ anion curves will be shifted downward relative to the neutral by $2.88 \mathrm{eV}$. In that case, attachment of an electron to the $\mathrm{C}=\mathrm{O} \pi^{*}$ orbital will be rendered exothermic. So, our data suggest that positively charged peptide samples can indeed attach low-energy electrons (e.g., as in ECD experiments) to carbonyl $\pi^{*}$ orbitals if positive sites are proximal enough to generate $>2.5 \mathrm{eV}$ of stabilizing Coulomb potential at the $\pi^{*}$ orbital (e.g., having a single protonated amine site within $5.8 \AA$ could generate such a potential).

Once an electron has attached to the $\mathrm{C}=\mathrm{O} \pi^{*}$ orbital, fragmentation of the bonds labeled 5, 7, or 8 in Figure 5 may be possible. From Figure 10, we can see that the paths leading to breaking bonds 5 or 8 will experience rather higher barriers (near where the $\pi^{*}$ and $\sigma^{*}$ diabatic curves cross, the barriers are 1.5 and $1.9 \mathrm{eV}$, respectively) and thus should be unlikely. Moreover, for these bond cleavages, the anion curve remains significantly above that of the neutral for all $R$ values, so the anion is subject to electron detachment throughout its fleeting existence. ${ }^{14}$

On the other hand, breaking bond 7, which generates the $\mathrm{C}-\mathrm{N}$ $\pi$-bond-forming process ${ }^{15}$ shown in Figure 8, has a lower barrier $(1.2 \mathrm{eV})$ and generates more thermodynamically stable products, so this bond cleavage is more likely to occur. Moreover, once the anion is formed by cleaving bond 7, it does not remain electronically metastable for all $R$ values. Soon after crossing (or tunneling through) the barrier on its surface, the anion becomes electronically stable and thus no longer susceptible to electron detachment. Of course, in the presence of a stabilizing Coulomb potential exceeding $2.5 \mathrm{eV}$, the anion is electronically stable throughout its evolution from $R$ near $1.5 \AA$ to larger values of $R$.

Because cleavage of bond 7 appears to be the best candidate for low-energy electron-induced cleavage, we repeated the series of calculations shown in Figure 10 (at the UHF level) using the correlated MP2 level of theory. In Figure 11 we show the neutral, $\pi^{*}$ anion, and $\sigma^{*}$ anion curves for this bond's cleavage.

Comparing Figures 10 and 11, we see that the energies of the anion states are differentially lowered relative to that of the neutral when we use a correlated approach, but not by so much as to render the findings obtained at the UHF level qualitatively incorrect.

The data of Figures 10 and 11 therefore suggest that the most likely bond cleavage to be observed is that of bond 7 , but this would require the incident electron to possess ca. $2.5 \mathrm{eV}$ of kinetic energy when no stabilizing Coulomb potential is operative. This energy permits both a near-vertical attachment to the $\pi^{*}$ anion and is adequate to access the anion-neutral crossing point. In the presence of Coulomb potentials greater 


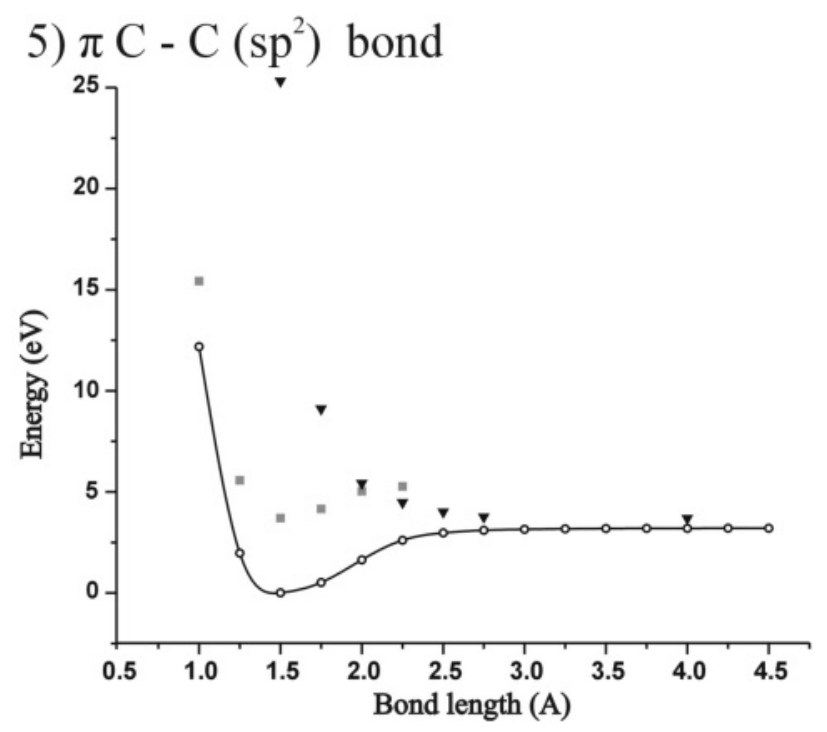

7) $\pi \mathrm{N}-\mathrm{C}$ bond

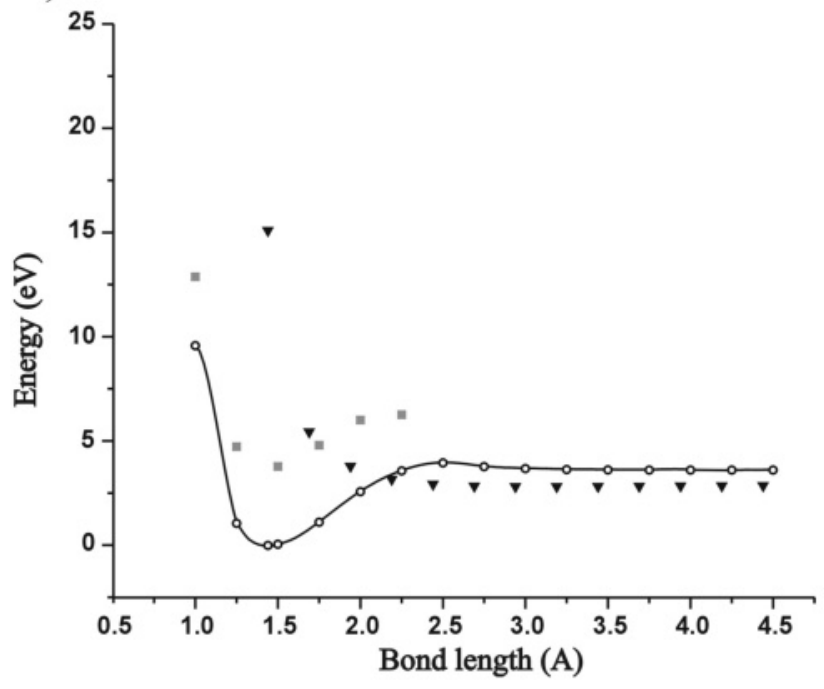

8) $\pi \mathrm{N}-\mathrm{C}$ bond

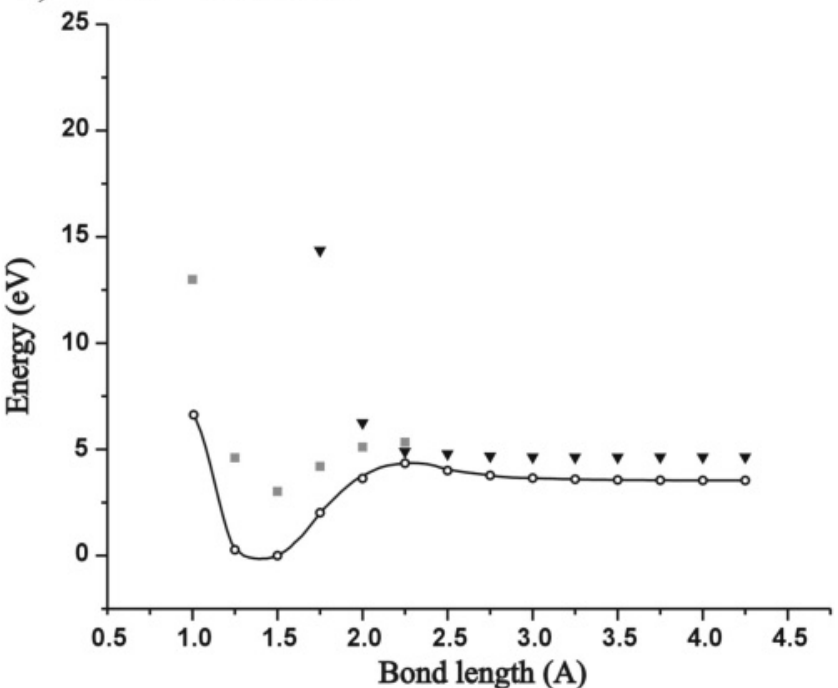

Figure 10. Energies of the neutral (open circles), $\pi^{*}$ anion (filled squares), and $\sigma^{*}$ anion (filled triangles) as functions of the lengths of the bonds labeled 5 (top), 7 (middle), and 8 (bottom) in Figure 5.

than $2.5 \mathrm{eV}$, the $\mathrm{C}=\mathrm{O} \pi^{*}$ orbital attachment and subsequent cleavage of bond 7 becomes exothermic and involves passing

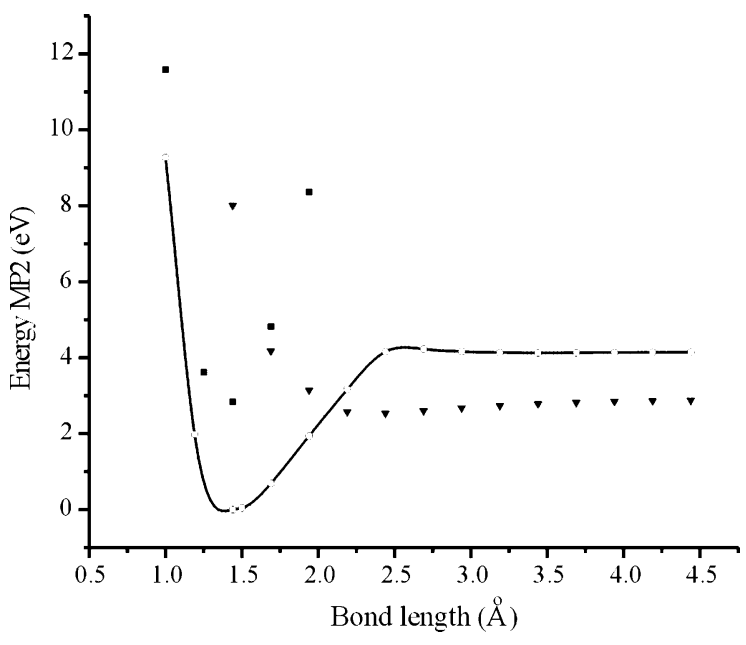

Figure 11. Energies of the neutral (open circles), $\pi^{*}$ anion (filled squares), and $\sigma^{*}$ anion (filled triangles) as functions of the lengths of the bond labeled 7 in Figure 5.

over or through a barrier. Indeed, as noted earlier, it is the kind of bond represented by bond 7 that is most commonly observed to cleave under ECD conditions in the laboratory where socalled $\mathrm{z}$ and $\mathrm{c}$ fragments are observed. The cleavage of bond 8 , which would produce what are termed $\mathrm{b}$ and $\mathrm{y}$ fragments, is observed when alternative fragmentation methods (e.g., collisional, electron impact, or infrared multiphoton absorption) are employed and fragmention at the carbonly $\mathrm{C}-\mathrm{N}$ bond occurs. ${ }^{12}$

\section{Summary}

Direct attachment of an electron to the $\sigma^{*}$ orbitals of eight different bonds within the model peptide molecule shown in Figure 5 has been examined using ab initio electronic structure methods. This particular care has been devoted to establishing a qualitatively correct description of the metastable anion states that arise with a device in which increased nuclear charges are used to stabilize the anion's energy. Moreover, indirect $\sigma$ bond cleavage, in which an electron first binds to a carbonyl $\mathrm{C}=\mathrm{O}$ $\pi^{*}$ orbital and subsequently breaks a nearby $\sigma$ bond, has also been examined.

It is found that direct low-energy electron attachment to and subsequent cleavage of any of the eight $\sigma$ bonds is not likely except for highly positively charged samples. Moreover, it is found that attachment to a $\mathrm{C}=\mathrm{O} \pi^{*}$ orbital followed by cleavage of the nearby $\mathrm{N}-\mathrm{C}_{\alpha}$ bond is the most likely outcome, although this process is vertically endothermic in the absence of Coulomb stabilization but rendered exothermic by $2.5 \mathrm{eV}$ or more of Coulomb potential energy. Interestingly, this bond 7 cleavage is what is seen most commonly in ECD experiments. ${ }^{11}$ So, the results presented here seem to offer good insight into one aspect of the ECD process, and they provide a means by which one can estimate (on the basis of a simple Coulomb energy formula) which bonds may be susceptible to cleavage by low-energy electron attachment.

Acknowledgment. This work was supported by NSF, grant nos. 9982420 and 0240387 to J.S. and by the Polish State Committee for Scientific Research (KBN), grant no. DS/83714-0137-4 to P.S. Significant computer time provided by the Center for High Performance Computing at the University of Utah and by the Academic Computer Center in Gdansk (TASK) is also gratefully acknowledged. Finally, we thank Prof. Robert Hudgins of York University for much helpful input and for nurturing our interest in the processes treated here. 


\section{References and Notes}

(1) Sawicka, A.; Skurski, P.; Hudgins, R. R.; Simons, J. J. Phys. Chem. $B$ 2003, 107, 13505-13511. Sobczyk, M.; Skurski, P.; Simons, J. Adv. Quantum Chem., in press. Sawicka, A.; Skurski, P.; Simons, J. Int. J. Quantum Chem., submitted 2004. Anusiewicz, I.; Sobczyk, M.; Skurski, P.; Simons, J. J. Phys. Chem. A, submitted 2004.

(2) Hudgins, R. R.; Håkansson, K.; Quinn, J. P.; Hendrickson, C. L.; Marshall, A. G. Unpublished, but in preparation. Although yet unpublished, these results have been presented in public by Hudgins, R. R.; Håkansson, K.; Quinn, J. P.; Hendrickson, C. L.; Marshall, A. G. Proceedings of the $50^{\text {th }}$ ASMS Conference on Mass Spectrometry and Allied Topics, Orlando, FL, June 2-6, 2002.

(3) Berges, J.; Kassab, E.; Conte, D.; Adjadj, E.; Houee-Levin, C. J. Phys. Chem. A 1997, 101, 7809-7817. Carles, S.; Leconte, F.; Schermann, J. P.; Desfrancois, C.; Xu, S.; Nilles, J. M.; Bowen, K. H.; Berges, J.; HoueeLevin, C. J. Phys. Chem. A 2001, 105, 5622-5626. Braida, B.; Hiberty, P. C. J. Phys. Chem. A 2003, 107, 4741-4747.

(4) Dezarnaud-Dandine, C.; Bournel, F.; Tronc, M.; Jones, D.; Modelli, A. J. Phys. B: At. Mol. Opt. Phys. 1998, 31, L497-L501. Modelli, A.; Jones, D.; Distefano, G.; Tronc, M. Chem. Phys. Lett. 1991, 181, 361-366.

(5) Of course, negatively charge groups can destabilize the $\sigma^{*}$ anion energy, but in this paper, we are dealing only with the effects of positively charged groups such as protonated amine sites in biomolecules.

(6) Zubarev, R.; Kruger, N.; Fridriksson, E. K.; Lewis, M. A.; Horn, D. M.; Carpenter, B. K.; McLafferty, F. W. J. Am. Chem. Soc. 1999, 121, 2857-2862. Zubarev, R. A.; Horn, D. M.; Fridriksson, E. K.; Kelleher, N. L.; Kruger, N. A.; Lewis, M. A.; Carpenter, B. K.; McLafferty, F. W. Anal. Chem. 2000, 72, 563-573. Syrstad, E. A.; Turecek, F. J. Phys. Chem. A 2001, 105, 11144-1115. Turecek, F.; Syrstad, E. A. J. Am. Chem. Soc. 2003 $125,3353-3369$.

(7) Kendall, R. A.; Dunning, T. H., Jr.; Harrison, R. J. J. Chem. Phys. 1992, 96, 6796

(8) Frisch, M. J.; Trucks, G. W.; Schlegel, H. B.; Scuseria, G. E.; Robb, M. A.; Cheeseman, J. R.; Montgomery, J. A., Jr.; Vreven, T.; Kudin, K. N.; Burant, J. C.; Millam, J. M.; Iyengar, S. S.; Tomasi, J.; Barone, V.; Mennucci, B.; Cossi, M.; Scalmani, G.; Rega, N.; Petersson, G. A.; Nakatsuji, H.; Hada, M.; Ehara, M.; Toyota, K.; Fukuda, R.; Hasegawa, J.; Ishida, M.; Nakajima, T.; Honda, Y.; Kitao, O.; Nakai, H.; Klene, M.; Li, X.; Knox, J. E.; Hratchian, H. P.; Cross, J. B.; Adamo, C.; Jaramillo, J.; Gomperts, R.; Stratmann, R. E.; Yazyev, O.; Austin, A. J.; Cammi, R.; Pomelli, C.; Ochterski, J. W.; Ayala, P. Y.; Morokuma, K.; Voth, G. A.; Salvador, P.; Dannenberg, J. J.; Zakrzewski, V. G.; Dapprich, S.; Daniels, A. D.; Strain, M. C.; Farkas, O.; Malick, D. K.; Rabuck, A. D.;
Raghavachari, K.; Foresman, J. B.; Ortiz, J. V.; Cui, Q.; Baboul, A. G.; Clifford, S.; Cioslowski, J.; Stefanov, B. B.; Liu, G.; Liashenko, A.; Piskorz, P.; Komaromi, I.; Martin, R. L.; Fox, D. J.; Keith, T.; Al-Laham, M. A.; Peng, C. Y.; Nanayakkara, A.; Challacombe, M.; Gill, P. M. W.; Johnson, B.; Chen, W.; Wong, M. W.; Gonzalez, C.; Pople, J. A. Gaussian 03 revision A.1; Gaussian, Inc.: Pittsburgh, PA, 2003.

(9) Ervin, K. M.; Anusiewicz, I.; Skurski, P.; Simons, J.; Lineberger, W. C. J. Phys. Chem. A 2003, 107, 8521-8529.

(10) It would require considerably more energy to break the $\mathrm{C}=\mathrm{O}$ double bond when this electron is attached. Lower energy pathways involve breaking some other $\sigma$ bond and leaving the excess electron on the carbonyl group's oxygen site (which has a large EA) to produce a $\mathrm{C}-\mathrm{O}^{-}$anion.

(11) We do not mean to imply that the electron capture mechanism studied here is the only means by which this pattern of bond cleavages could occur. It is indeed possible for a $\mathrm{H}$ atom, formed elsewhere in the molecule, to attack the $\mathrm{C}=\mathrm{O} \pi$ bond to form a $\mathrm{C}-\mathrm{OH}$ radical, after which the $\mathrm{N}-\mathrm{C}_{\alpha}$ bond could break and allow a new $\mathrm{N}-\mathrm{C} \pi$ bond to form. This would then produce the same fragmentation pattern. We only suggest here that electron capture into the $\pi^{*}$ orbital offers one means by which this same fragmentation might occur. We also note that subsequent proton transfer to the $-\mathrm{CO}^{-}$center from elsewhere in the parent ion would generate the same $-\mathrm{C}-\mathrm{OH}$ unit as observed in the $\mathrm{H}$ atom transfer process just mentioned.

(12) Kruger, N. A.; Zubarev, R. A.; Carpenter, B. K.; Kelleher, N. L.; Horn, D. M.; McLafferty, F. W. Int. J. Mass Spectrom. 1999, 182/183, $1-5$.

(13) The electronic mixing that couples the $\pi^{*}$ and $\sigma^{*}$ diabatic configurations of the anion give rise to lower and upper adiabatic anion surfaces. On the lower adiabatic surface, which we denote $\pi^{*} / \sigma^{*}$, is where the anion's motion is assumed to occur.

(14) The metastability of the anion persists until the bond is very stretched, at which time the anion can become electronically stable. Such stability is not shown in our potential plots, because the anion energies were evaluated vertically at the geometry of the anion. If the anion geometry were allowed to relax to become energetically optimal, the anion energy would drop slightly below that of the neutral at very long $R$-values

(15) When the geometry of the anion is allowed to relax to achieve optimal energy (but for a fixed value of the length of bond 7), the $\mathrm{C}=\mathrm{O}$ bond is found to lengthen (relative to its value in the neutral compound) by $0.04 \AA$, while the $\mathrm{C}-\mathrm{N}$ bond shrinks by $0.04 \AA$. This is an indication of the formation of the $\mathrm{C}=\mathrm{N} \pi^{*}$ bond that accompanies the process shown in Figure 8. 